\title{
The mazEF toxin-antitoxin system as a novel antibacterial target in Acinetobacter baumannii
}

\author{
Sobhan Ghafourian ${ }^{1,2} /{ }^{+}$, Liam Good ${ }^{3}$, Zamberi Sekawi', Rukman Awang Hamat', \\ Sara Soheili', Nourkhoda Sadeghifard ${ }^{2}$, Vasanthakumari Neela ${ }^{1 /+}$
}

\begin{abstract}
'Department of Medical Microbiology and Parasitology, Faculty of Medicine and Health Sciences, Universiti Putra Malaysia, Serdang, Selangor, Malaysia ${ }^{2}$ Clinical Microbiology Research Center, Ilam University of Medical Sciences, Ilam, Iran ${ }^{3}$ Pathology and Pathogen Biology, Royal Veterinary College, University of London, London, United Kingdom
\end{abstract}

Although analysis of toxin-antitoxin (TA) systems can be instructive, to date, there is no information on the prevalence and identity of TA systems based on a large panel of Acinetobacter baumannii clinical isolates. The aim of the current study was to screen for functional TA systems among clinical isolates of A. baumannii and to identify the systems' locations. For this purpose, we screened 85 A. baumannii isolates collected from different clinical sources for the presence of the mazEF, relBE and higBA TA genes. The results revealed that the genes coding for the mazEF TA system were commonly present in all clinical isolates of A. baumannii. Reverse transcriptase-polymerase chain reaction analysis showed that transcripts were produced in the clinical isolates. Our findings showed that TA genes are prevalent, harboured by chromosomes and transcribed within A. baumannii. Hence, activation of the toxin proteins in the mazEF TA system should be investigated further as an effective antibacterial strategy against this bacterium.

Key words: toxin-antitoxin systems - Acinetobacter baumannii - antibacterial target

The use of antibiotics for the treatment of infection in humans and animals over the past decades has led to the evolution of multiple drug resistance in microbes. Resistance to antibiotics develops through mutations of target sites or the acquisition of antibiotic-resistant genes from other pathogens. To date, multiple drug resistance has been the greatest challenge in the management of infectious diseases. Although studies on the development of new antibiotics are rapidly progressing, the identification of new drug targets in microbes is also very important for effective infection control. Currently, resistance to antimicrobial agents seems to be a problematic issue worldwide. Thus, finding new antibiotics as a strategy to continue antibiotic therapy seems urgent (Davies \& Webb 1998, Schlessinger \& Eisenstein 1999).

A toxin-antitoxin (TA) system is a set of two or more closely linked genes that together encode both a protein "poison" and a corresponding "antidote". TA systems were first described in 1982 (Ghafourian et al. 2014). Detailed insights into their functions and mechanisms of action have been gained over the last two decades and have led to several interesting conclusions about the importance of such systems for bacterial physiology. TA systems consist of a toxin and an antitoxin protein; when expressed, the toxin kills the bacterium. In typi-

doi: $10.1590 / 0074-0276130601$

+Corresponding author: sobhan.ghafurian@gmail.com,

neela2000@hotmail.com

Received 29 December 2013

Accepted 7 May 2014 cal bacterial physiology, the antitoxin binds to the toxin and neutralises it, hence preventing the suicide of the bacterium. The toxin protein is always stable, whereas the antitoxin is unstable. The unstable nature of the antitoxin makes it highly susceptible to proteolytic degradation. When the antitoxin is degraded or not functional, the toxin produced kills the bacterium, which is known as programmed cell death (Cherny 2007). Thus, this system has recently gained attention, especially as a target for antimicrobial agents. However, the presence and type of TA system vary from bacterium to bacterium. TA systems can be either plasmid encoded or chromosomally mediated. In plasmid-mediated systems, if the plasmid is lost during cell division, the antitoxin protein rapidly degrades and is not replenished. The stable toxin is thus released, killing the bacterial cell (Gerdes 1986, Gerdes et al. 2005).

In the present investigation, we aimed to screen for different TA systems in Acinetobacter baumannii. Acinetobacter species are top ranking among nosocomial pathogens with multiple drug-resistant strains. These species cause various nosocomial infections, such as bacteraemia, urinary tract infection, secondary meningitis, surgical site infection and nosocomial and ventilator-associated pneumonia (Go et al. 1994). The management of Acinetobacter infections is always challenging, especially in intensive care units, with many mortality reports. Identifying the types of TA systems, whether they are functional and their location in A. baumannii will provide a fundamental understanding of their application as antibiotic targets.

Eighty-five unique multidrug-resistant $A$. baumannii isolates isolated from different clinical sources in Malaysia were investigated. All isolates were subjected to amplification of the mazEF, relBE and higBA TA genes within chromosomal and plasmid DNA using primers designed based on the corresponding gene sequence 
in the gene bank (Table I). Polymerase chain reaction (PCR) amplification was performed in a DNA thermal cycler (Bio-Rad) using the amplification parameters described previously (Moritz \& Hergenrother 2007), with a lowering of the annealing temperature to $58^{\circ} \mathrm{C}$. The PCR-amplified products were analysed by agarose gel electrophoresis. To identify whether the TA systems were encoded in plasmid or chromosomal DNA, PCR amplification for all isolates was conducted individually with plasmid and chromosomal DNA. To assess functionality, reverse transcriptase (RT)-PCR was performed using the SuperScript One-Step RT-PCR System with Platinum Taq (Invitrogen). The reverse transcription and PCR amplification were conducted in a DNA thermal cycler (Bio-Rad) under the reaction conditions described previously (Moritz \& Hergenrother 2007), using the primers used for the conventional PCR described above. The RT-PCR amplification products were analysed by agarose gel electrophoresis.
The results of screening chromosomal DNA for the presence of different TA loci showed that all $\mathrm{A}$. baumannii isolates $(85 / 85,100 \%)$ were positive for $m a z E F$ and that relBE was found in $88.2 \%$ of isolates $(75 / 85)$, whereas $\operatorname{hig} B A(4 / 85,4.7 \%)$ was less prevalent (Table II). PCR analysis of plasmid DNA did not yield any positive amplification, indicating that in $A$. baumannii, the screened TA systems were chromosomally mediated. To confirm the functionality of the TA systems, all clinical isolates containing TA systems were subjected to RTPCR. RNA from all isolates was collected and subjected to cDNA synthesis. PCR was then performed to confirm the functionality of the TA systems. The positive results confirmed the functionality of the TA systems.

TA system transcripts were detected from the total RNA of all A. baumannii strains probed by RT-PCR. The results of the RT-PCR strongly indicated that the TA systems are functional in all A. baumannii. To control for the contamination of any reaction with DNA, a

TABLE I

Gene-specific primers used for polymerase chain reaction (PCR) and reverse transcriptase-PCR analysis

\begin{tabular}{lcc}
\hline Toxin-antitoxin systems & Primer sequences & Length \\
\hline \multirow{2}{*}{ mazEF } & Forward: ACCTTCGAAGGAACTACGTCAGTAG & $436 \mathrm{bp}$ \\
& Reverse: ATAGGCGAACATGCAAGAAAAGGCAGC & $578 \mathrm{bp}$ \\
relBE & Forward: ATGAAGTGAACGGTCAACAATA & \\
& Reverse: ACAGACCTCGGAAAGTGGTCG \\
higA & Forward: AGCACATCCGTACGATCTACTGC-3 & $440 \mathrm{bp}$ \\
& Reverse: TGCACTCCTGCGATGCGGCGAA & \\
\hline
\end{tabular}

TABLE II

Prevalence of different toxin-antitoxin loci in Acinetobacter baumannii

\begin{tabular}{|c|c|c|c|c|c|c|}
\hline Isolates & $m a z E F$ & $\operatorname{hig} B A$ & relBE & $\begin{array}{c}\text { RT-PCR } \\
\text { results of } m a z E F\end{array}$ & $\begin{array}{c}\text { RT-PCR } \\
\text { results of higBA }\end{array}$ & $\begin{array}{c}\text { RT-PCR } \\
\text { results of relBE }\end{array}$ \\
\hline 1 & + & - & - & + & - & - \\
\hline 2 & + & - & + & + & - & + \\
\hline 3 & + & - & + & + & - & + \\
\hline 4 & + & - & + & + & - & + \\
\hline 5 & + & - & + & + & - & + \\
\hline 6 & + & - & + & + & - & + \\
\hline 7 & + & - & + & + & - & + \\
\hline 8 & + & - & + & + & - & + \\
\hline 9 & + & - & - & + & - & - \\
\hline 10 & + & - & + & + & - & + \\
\hline 11 & + & - & + & + & - & + \\
\hline 12 & + & + & + & + & + & + \\
\hline 13 & + & - & + & + & - & + \\
\hline 14 & + & - & + & + & - & + \\
\hline 15 & + & - & + & + & - & + \\
\hline 16 & + & - & + & + & - & + \\
\hline 17 & + & - & - & + & - & - \\
\hline 18 & + & - & - & + & - & - \\
\hline
\end{tabular}




\begin{tabular}{|c|c|c|c|c|c|c|}
\hline Isolates & $m a z E F$ & $\operatorname{hig} B A$ & relBE & $\begin{array}{c}\text { RT-PCR } \\
\text { results of } m a z E F\end{array}$ & $\begin{array}{c}\text { RT-PCR } \\
\text { results of higBA }\end{array}$ & $\begin{array}{c}\text { RT-PCR } \\
\text { results of relBE }\end{array}$ \\
\hline 19 & + & - & + & + & - & + \\
\hline 20 & + & - & + & + & - & + \\
\hline 21 & + & + & + & + & + & + \\
\hline 22 & + & + & + & + & + & + \\
\hline 23 & + & - & + & + & - & + \\
\hline 24 & + & - & + & + & - & + \\
\hline 25 & + & - & + & + & - & + \\
\hline 26 & + & - & + & + & - & + \\
\hline 27 & + & - & + & + & - & + \\
\hline 28 & + & - & + & + & - & + \\
\hline 29 & + & - & + & + & - & + \\
\hline 30 & + & - & + & + & - & + \\
\hline 31 & + & - & + & + & - & + \\
\hline 32 & + & - & - & + & - & - \\
\hline 33 & + & - & + & + & - & + \\
\hline 34 & + & - & + & + & - & + \\
\hline 35 & + & - & + & + & - & + \\
\hline 36 & + & - & + & + & - & + \\
\hline 37 & + & - & + & + & - & + \\
\hline 38 & + & - & + & + & - & + \\
\hline 39 & + & - & + & + & - & + \\
\hline 40 & + & - & - & + & - & - \\
\hline 41 & + & - & + & + & - & + \\
\hline 42 & + & - & + & + & - & + \\
\hline 43 & + & - & + & + & - & + \\
\hline 44 & + & - & + & + & - & + \\
\hline 45 & + & + & + & + & + & + \\
\hline 46 & + & - & + & + & - & + \\
\hline 47 & + & - & - & + & - & - \\
\hline 48 & + & - & - & + & - & - \\
\hline 49 & + & - & + & + & - & + \\
\hline 50 & + & - & + & + & - & + \\
\hline
\end{tabular}

reaction without the RT enzyme, but including the other materials, was used. The negative results confirmed that no contamination of the DNA had occurred. In a study on Escherichia coli and the cyanobacterium Anabaena by Ning et al. (2013), similar results were obtained, which showed the functionality of the mazEF TA system. Moritz and Hergenrother (2007) demonstrated the functionality of $m a z E F$ using RT-PCR by evaluating 10 vancomycin resistance enterococcus (VRE) isolates and found that $m a z E F$ TA loci were transcribed in all 10 VRE isolates. We used PCR as a tool to screen for different TA systems in A. baumannii and found that mazEF TA loci were frequently present.

Identifying TA systems in clinical isolates is important for validating these systems as antibacterial targets. In the current study, RT-PCR analysis was performed to determine whether the TA systems were transcribed. RTPCR showed that the $m a z E F$, higBA and relBE TA systems were functional in all strains positive for TA loci.

It has been suggested that by activating TA systems (disrupting antitoxin or causing increased toxin expres- sion), it is possible to find a new sensitive target in $A$. baumannii (Engelberg-Kulka et al. 2004, Gerdes et al. 2005, Williams \& Hergenrother 2008). Unfortunately, no previous study has examined the prevalence of TA systems in a large panel of clinical isolates of $A$. baumannii. In addition, this is the first time that the $\operatorname{maz} E F, \operatorname{hig} B A$ and relBE transcripts have been shown to be present in A. baumannii and one of the few examples demonstrating the transcription of any TA loci in a clinical isolate.

The current research indicated that TA systems are prevalent, harboured by chromosomes and transcribed in clinical isolates of A. baumannii.

\section{REFERENCES}

Cherny I 2007. Structural and thermodynamic characterization of the Escherichia coli RelBE toxin-antitoxin system: indication for a functional role of differential stability. Biochemistry 46: 12152-12163.

Davies J, Webb V 1998. Antibiotic resistance in bacteria. In RM Krause (ed.), Emerging infections: biomedical research reports, Academic Press, San Diego, p. 239-273. 
Engelberg-Kulka H, Sat B, Reches M, Amitai S, Hazan R 2004. Bacterial programmed cell death systems as targets for antibiotics. Trends Microbiol 12: 66-71.

Gerdes K 1986. Unique type of plasmid maintenance function: postsegregational killing of plasmid-free cells. Proc Natl Acad Sci USA 83: 3116-3120.

Gerdes K, Christensen SK, Lobner-Olesen A 2005. Prokaryotic toxinantitoxin stress response loci. Nat Rev Microbiol 3: 371-382.

Ghafourian S, Raftari M, Sadeghifard N, Sekawi Z 2014. Toxin-antitoxin systems: classification, biological function and application in biotechnology. Curr Issues Mol Biol 16: 9-14.

Go ES, Urban C, Burns J, Kreiswirth B, Eisner W, MarianoN 1994. Clinical and molecular epidemiology of acinetobacter infections sensi- tive only to polymyxin B and sulbactam. Lancet 12: 1329-1332.

Moritz EM, Hergenrother PJ 2007. Toxin-antitoxin systems are ubiquitous and plasmid-encoded in vancomycin-resistant enterococci. Proc Natl Acad Sci USA 104: 311-316.

Ning D, Jiang Y, Liu Z, Xu Q 2013. Characterization of a chromosomal type II toxin-antitoxin system mazEaFa in the cyanobacterium Anabaena sp. PCC 7120. PLoS ONE 8: e56035.

Schlessinger D, Eisenstein B 1999. Biological basis for antibacterial action. In M Schaechter, NC Engleberg, B Eisenstein, G Medoff (eds.), Biological basis for antibacterial action, 3rd ed., Williams \& Wilkins, New York, p. 52-60.

Williams JJ, Hergenrother PJ 2008. Exposing plasmids as the Achilles' heel of drug-resistant bacteria. Curr Opin Chem Biol 12: 389-399. 